\title{
Programa de reabilitação neuropsicológica da memória aplicada à demência: um estudo não controlado intrasujeitos ${ }^{1}$
}

\author{
Neuropsychological rehabilitation of memory \\ applied to dementia: an intra-subject \\ non-controlled study
}

\author{
Sérgio Leme DA-SILVA ${ }^{2}$ \\ Danilo Assis PEREIRA ${ }^{3}$ \\ Fabrízio VELOSO ${ }^{4}$ \\ Corina Elizabeth SATLER ${ }^{4}$ \\ Adriana ARANTES ${ }^{5}$ \\ Renato Maia GUIMARÃES ${ }^{4}$
}

\begin{abstract}
Resumo
Neste estudo não controlado intrassujeitos, 21 idosos com Alzheimer ou outras demências participaram de um Programa de Reabilitação Neuropsicológica, com oficinas de jardinagem e pistas coloridas. Após o programa, houve aumento nos escores dos seguintes testes: Miniexame do Estado Mental $(Z=-1,98, p<0,05)$; Subteste Verbal de Semelhanças da Escala Wechsler $[(Z=-2,09) p<0,05]$ e Subteste de Aprendizagem de Pares de Fácil Associação para Evocação Tardia da Escala de Memória de Wechsler $[(Z=-2,07) p<0,05]$. Paralelamente, observou-se redução dos escores de depressão na Escala de Depressão Geriátrica de Yesavage $[(Z=-3,02) p<0,00]$. Foi demonstrado ainda que essa redução estava associada à aprendizagem de pistas contextuais (reminiscências e sinalizadores) e ao tratamento com anticolinesterásicos administrados por 4 ou 12 semanas $[(Z=-2,31)$ $p<0,02]$. Ressalta-se, entretanto, que o mesmo não ocorreu com participantes submetidos ao tratamento de 30 semanas $[(Z=-2,21) p<0,02]$.
\end{abstract}

Unitermos: Demência. Depressão. Idoso. Memória. Reabilitação neuropsicológica.

\begin{abstract}
In this intra-subject non-controlled study, twenty-one elderly patients with Alzheimer Disease or other forms of dementia participated in a neuropsychological rehabilitation program involving gardening and colored cues. At theend of the neuropsychological rehabilitation program,
\end{abstract}

rQver

1 Apoio Financiadora de Estudos e Projetos do Ministério de Ciência e Tecnologia - Cód. FINEP - 0106032800 - Processo FUB-UnB Cód. 05900 Convênio ref. $2261 / 05$.

2 Universidade de Brasília, Instituto de Psicologia, Departamento de Processos Psicológicos Básicos. Campus Universitário Darcy Ribeiro, Asa Norte, 70910-900, Brasília, DF, Brasil. Correspondência para/Correspondence to: S.L. DA-SILVA. E-mail: <leme@unb.br>.

3 Instituto Brasileiro de Neuropsicologia e Ciências Cognitivas. Brasília, DF, Brasil.

- Hospital Universitário de Brasília, Centro de Medicina do Idoso. Brasília, DF, Brasil.

s Hospital Universitário de Brasília, Serviço de Pedagogia. Brasília, DF, Brasil.

Agradecimentos: Iraci Gonçalves Guimarães, Fernando de Barros, Juliana Lima Quintas, Mariana Balduíno, Josely Gomes, Eric-Karl H. Diethelm, Fabíola Krystina S. Celestino, Lucia Inês de Araújo, e as Equipes de Saúde do CMI. 
improved scores were noted in the following tests: Mini-Mental State Examination ( $Z=-1.98, p<0.05)$; Wechsler Verbal Performance Scale subtest [ $(Z=-2.09) p<0.05]$; Wechsler Memory Scale (Associated Pairs with Delayed Recall) $[(Z=-2.07) p<0.05]$ and in the Yesavage Geriatric Depression Scale [ $(Z=-3.02) p<0.00]$. It was also demonstrated that patients who learnt the contextual cues (reminiscences and flags) saw a significant reduction in the Geriatric Depression Scale. Depression Scale scores were lower in those who correctly free-recalled this association $(F=2.12, p=0.14)$. Use of anticholinesterase drugs in the $4^{\text {th }}$ and $12^{\text {th }}$ weeks was associated with a reduction in the Geriatric Depression Scale, but not for those in the $30^{\text {th }}$ week following the neuropsychological rehabilitation program.

Uniterms: Dementia. Depression. Aged. Memory. Neuropsychological rehabilitation.

Existem inúmeras dificuldades a serem consideradas no estabelecimento de formas de Programas de Reabilitação Neuropsicológica (PRN) que funcionem simultaneamente como terapêutica para idosos portadores de demência e como alternativa preventiva contra o declínio cognitivo e a depressão.

A primeira dessas dificuldades consiste em estabelecer uma meta do PRN, em harmonia com a prática clínica de estimulação cognitiva e as necessidades do quadro sintomático em questão. O objetivo dessa harmonia é gerar a promoção de algum ganho, seja ele cognitivo, comportamental, emocional, social, educativo ou adaptativo. Entretanto, qualquer que seja o objetivo do PRN, sua construção deve enfocar sempre a melhora da qualidade de vida e, por conseguinte, prevenir, tratar ou contornar as características sintomáticas dos transtornos cognitivos e afetivos associados ao envelhecimento, sem deixar de considerar as particularidades da própria doença e do indivíduo (Prigatano, 1995; Wilson, 1997). Assim, devem ser individualmente considerados os fatores de gênero, idade e influências ambientais, pois eles podem alterar o ritmo dos estados sintomáticos da Doença de Alzheimer (DA) (Abrisqueta-Gomez et al. 2002; Da-Silva, 2004).

O PRN deve estar associado a uma Avaliação Neuropsicológica (AN) sensível para reconhecer diferentes perfis cognitivos de demências e seus estágios, principalmente o inicial e o intermediário, e ainda promover estimulações cognitivas e comportamentais preventivas que possam lentificar a evolução da doença (Lincoln \& Nair, 2008; Sohlberg \& Mateer, 2001).

\section{A reaplicabilidade e validade de um PRN}

Outra dificuldade em estabelecer a forma mais adequada de um PRN está na reaplicabilidade e validade do modelo (Lincoln \& Nair, 2008). Os mais adequados redução ou a compensação das dificuldades, ou, ainda, dos problemas verificados na execução de atividades cognitivas e que possam ser medidos e conceituados. Dentre esses modelos, os mais reconhecidos são as estratégias compensatórias, a orientação para a realidade, as reminiscências, os recursos mnemônicos, a mnemotécnica, a aprendizagem sem erro e a psicoeducação dos cuidadores (De Vreese, Néri, Fioravanti, Belloi \&Zanetti, 2001; Wilson 1987, 1997; Wilson \& Moffat, 1992).

Os limites dessas técnicas, porém, devem ser considerados em relação às especificidades da doença, da população atendida, dos cuidadores, do suporte social, da instituição e da própria equipe.

A técnica de estratégias compensatórias, por exemplo, que compreende o uso de apoios externos - como gravadores, agendas, sinalizadores, cartazes etc.-, produz efeitos benéficos quando os pacientes estão em fase inicial, e menos na fase intermediária, dependendo do auxílio do cuidador e do terapeuta (Bourgeois, 1990).

A técnica de orientação de realidade é o treino sistemático de informações presentes e contínuas, amparadas por estímulos ambientais de orientação espacial e temporal. Facilita a sociabilidade e pode ser orientada por apoios externos. Essa técnica vem sendo muito utilizada em idosos (Vaisman, K.M.H. Almeida \& O.P. Almeida, 1997).

A técnica das reminiscências consiste em utilizar diversos materiais de contexto autobiográfico (fotos, músicas, roupas etc.), além das próprias histórias remotas que fazem parte do acervo mnemônico preservado do paciente. Essa técnica estimula a socialização e a integração no grupo terapêutico e é habitualmente utilizada em grupo e em pacientes confusos, como também em idosos demenciados (Fraser, 1992).

Os recursos mnemônicos compreendem os apoios verbais e visuais associados ao aprendizado. A dificuldade para seu uso está na capacidade de o 
indivíduo amnésico utilizá-lo por iniciativa própria. Entretanto, esses recursos produzem resultados mais eficientes no armazenamento de uma nova informação, se comparados à repetição (Wilson, 1987; 2009).

A mnemotécnica foi proposta como um aprimoramento dos recursos mnemônicos, ao aplicar uma associação ou combinação de informações mentais para serem recordadas em conjunto. Podem-se utilizar histórias que se conectem a materiais a serem recordados, palavras sem sentido treinadas com um objeto a serem recordadas, ou ainda nomes ou rostos ligados a uma característica da pessoa (Wilson, 1997).

O aprendizado sem erros é o reforço das respostas corretas. Nessa técnica, não há acentuação ou reforço das respostas com erro (Wilson, 2009). Para uma boa execução desse aprendizado, é necessária a realização de um programa que impeça o indivíduo amnésico de emitir "respostas-palpites" ou em forma de tentativa e erro. Essa técnica tem como base os estudos com memória implícita, em que o indivíduo é capaz de aprender e desempenhar uma habilidade, mesmo que não tenha capacidade de lembrá-la (Brooks \& Baddeley, 1976).

A orientação aos familiares sobre o tratamento, denominada psicoeducação de cuidadores, é outra técnica importante. Vários pesquisadores têm enfatizado que a falta de informação sobre a doença e sua evolução, como também os encargos estressantes e emocionais, podem gerar impacto desestruturante sobre a vida do cuidador (Garrido \& Almeida, 1999). Cuidadores bem orientados e estruturados emocionalmente têm mais possibilidades de dar continuidade ao uso das técnicas de reabilitação cognitiva e, por conseguinte, contribuem para a melhoria da qualidade de vida, tanto do paciente quanto sua própria (Sohlberg \& Mateer, 2001).

Com o objetivo de reforçar a validade ou confiança no PRN impõem-se o desenvolvimento de um modelo baseado nos conceitos da neurociência cognitiva, como os sistemas de memória (operacional, explícita, episódica, semântica e implícita), além daqueles associados a uma possibilidade de incrementar ou estimular memórias, como repetição, pré-ativação, ativação espraiada de rede semântica, valência emocional, pensamento associativo categórico (Baddeley, 1998; Squire, 1987; Wilson, 2009). O PRN, através de seus resultados, sugere fundamentos sobre a efetividade da estimulação das capacidades cognitivas mnemônicas residuais ou preservadas, além de compreender por quais meios tais sistemas, mesmo que prejudicados, possam estar de alguma forma ativos.

\section{O impacto do PRN sobre a plasticidade cerebral}

A plasticidade cerebral é uma das questões centrais das neurociências. É nesse cenário que se estabelece a relação de reciprocidade entre o organismo e o meio ambiente, e, por conseguinte, a possibilidade da aplicação de um PRN gerar impacto plástico cerebral (Kolb \& Gibb, 2008).

Entretanto, a questão se torna "uma luta contra a maré" quando se sumarizam estudos anatomopatológicos de pacientes de DA com quadros patológicos de placas neuríticas, novelos fibrilares, degeneração de neurônios do hipocampo, atrofia cortical difusa, dilatação ventricular, além de alterações neuroquímicas primordiais no sistema colinérgico e mudanças no sistema dopaminérgico e serotonérgico, entre outras desordens (Grady, Haxby, Horwitz, Berg \& Rapoport, 1988).

Em contrapartida, a razão primordial em se acreditar na existência da plasticidade cerebral está na forma diferente de olhar o próprio desenvolvimento natural, isto é, em conhecer a ontogenia do cérebro, passível de influências advindas de modificações no ambiente e no modo de vida. Nesse sentido, pode-se citar o trabalho clássico de Rosenzweig, Krech, Bennette Diamond (1962) envolvendo a criação de roedores em gaiolas-viveiro, aos quais foi oferecida grande variedade de estímulos, como objetos de formas diferentes, espelhos, rodas de atividade, escadas, espaço maior, além de diferentes possibilidades para conseguir alimento. Outro grupo de roedores foi criado em gaiolas sem nenhum estímulo, a não ser a oferta de comida e água. Os autores demonstraram, através de técnicas histológicas, que animais de diferentes idades, criados em ambientes enriquecidos, apresentavam aumento na espessura das camadas do córtex visual, no tamanho dos núcleos e corpos neuronais, no número de sinapses e na área das zonas de contato sináptico, no número de dendritos e de espinhas dendríticas, no volume e no peso cerebral, além de alterações nos neurotransmisso-res. Jankowsky et al. (2005), utilizando duas linhagens de ratos, modificados geneticamente durante 10 gerações para obtenção de 
animais sensíveis à alta produção de placas amiloides, demonstraram que esses animais, quando expostos a enriquecimento ambiental, eliminam seus défices cognitivos em tarefas natatórias onde o objetivo é encontrar a plataforma submersa, sugerindo que esse resultado é pertinente com teorias de reserva cognitiva em humanos portadores de Alzheimer.

Joca, Padovan e Guimarães (2003) discutiram as influências de fatores estressantes sobre a depressão e a plasticidade do hipocampo, dando consistência à hipótese de que o estresse pode causar alterações plásticas no hipocampo. Tais alterações incluem remodelação dendrítica e inibição de neurogênese, revelando-se mais ativas em pacientes com resistência a antidepressivos e em animais mais sensíveis a modelos de depressão.

Esses trabalhos vêm subsidiando a hipótese da existência de interações plásticas e recíprocas entre o organismo e o ambiente, reforçando a ideia de que PRN em situações ambientais possuem a capacidade de estimular plasticamente a base biológica da memória, e isso com ou sem o auxílio de terapêuticas farmacológicas, o que, todavia, não significa que estas sejam consideradas dispensáveis (Kolb \& Gibb, 2008).

\section{Impactos de PRN em idosos}

Estudos randomizados controlados mostraram que o treino cognitivo pode melhorar a memória, a atenção, o humor e o bem-estar psíquico, mas o impacto na vida diária e a duração desses efeitos ainda são desconhecidos (Belleville, Gilbert \& Fontaine, 2006; Rapp, Brene \& Marsh 2002; Rozzini, Costardi \& Vicini-Chilovi, 2006; Talassi et al., 2007).

De acordo com Kurz, Pohl, Ramsenthaler e Sorg (2009), a melhoria na Atividade de Vida Diária (AVD) e na memória de pacientes com declínio cognitivo leve estava relacionada à redução de sintomas depressivos. No grupo de intervenção, os pacientes com demência leve tiveram uma melhora significativa da memória episódica verbal e uma ligeira redução no escore de depressão, mas nenhuma mudança na AVD. Ainda, essa pesquisa confirmou os resultados anteriores de estudos controlados sobre os efeitos do treinamento cognitivo

232 (Belleville et al., 2006; Cipriani, Bianchetti \& Trabucchi,
2006; Rozzini et al., 2006; Talassi et al., 2007) e de um recente estudo não controlado sobre o potencial cognitivo na reabilitação de pacientes com prejuízo cognitivo leve (Londos et al., 2008).

Num estudo piloto não controlado, Da-Silva (2004) relatou benefícios diferenciados cognitivos e de humor, advindos de um PRN em oito idosos demenciados.

Esses estudos demonstraram o impacto dos PRN sobre o desempenho cognitivo, a velocidade de processamento de informação, a autoavaliação da capacidade funcional e o bem-estar subjetivo. Além disso, observaram que a melhoria na AVD e na memória estava relacionada à redução no escore de depressão.

Este trabalho analisa os efeitos de um PRN em pacientes demenciados, através de alterações emocionais e cognitivas, refletidas na avaliação neuropsicológica e no desempenho de tarefas aprendidas.

\section{Método}

Este PRN foi composto de AN prévias e posteriores, Oficina para Estimulação da Memória (OEM), mediante o uso de jardinagem e pistas coloridas, bem como de sessões de Grupo Psicoeducativo (GPE), dirigidas aos cuidadores e familiares.

Ademais, este PRN foi construído principalmente com base nas ideias de reabilitação da memória de Wilson (1987; 2009), e de acordo com a revisão de Rothi acerca do Modelo de Fases de Robey sobre pesquisas em reabilitação neuropsicológica (Rodrigues \& Rothi, 2008). Trata-se de estudo clínico exploratório, envolvendo população definida, pequena e não controlada, mediante análise experimental intrassujeitos.

O método aplicado utilizou as seguintes premissas: 1) trabalho terapêutico com grupo de até oito idosos; 2) conscientização do grupo acerca da dificuldade de memória, mediante explicitação, em todas as sessões, de que o fenômeno era comum a todos e da importância de estimular a memória para diminuir os erros; 3) administração e uso da capacidade preservada e do potencial de resíduo cognitivo, ligado principalmente às reminiscências históricas de cada participante; 4) administração e uso de estratégias compensatórias, por meio do auxílio constante de apoios externos, como 
os sinalizadores (cores, aventais, vasos e plantas); 5) vigilância quanto à compreensão e atenção para com as tarefas; 6) flexibilização do tempo na execução das tarefas; 7) uso de repetições; 8) uso de associações semânticas, reminiscências e significados emocionais ligados à escolha da cor a ser utilizada na oficina de estimulação da memória; 9) elaboração sistemática de planejamento pedagógico da reabilitação neuropsicológica (2 sessões de avaliação neuropsicológica e 6 sessões de oficina, totalizando 8 sessões padronizadas); 10) aplicação de avaliações neuropsicológicas prévias e posteriores, na primeira e na última sessão do PRN; 11) registro e recuperação do desempenho dos idosos nas 6 sessões de oficina; na tarefa de evocação das cores que serviram como Sinalizadores (SI); na evocação do contexto histórico de escolha dos SI; no registro do tipo de resposta: resposta livre sem ajuda, resposta com ajuda contextual da reminiscência, resposta de não recordação; 12) realização de atividades, a cada sessão de grupo psicoeducativo, dirigidas aos cuidadores, com apresentação de feedback sobre o desempenho dos idosos nas oficinas, além de orientações referentes à doença, tratamento e evolução do quadro.

O presente estudo é parte do projeto "Programa Presencial e Virtual de Gerontologia Domiciliar, Avaliação e Reabilitação Neuropsicológica - Projeto GEDARNI", que contou com o apoio financeiro da Financiadora de Estudos e Projetos (FINEP). Foi aprovada previamente a sua aplicação pelo Comitê de Ética em Pesquisa da Faculdade de Ciências da Saúde da Universidade de Brasília (CEP/FS), sob o protocolo n 109/2007, em 17 de dezembro de 2007. Todos os participantes assinaram o termo de consentimento livre e esclarecido, antes de sua inclusão na amostra.

\section{Participantes}

Todos os participantes do PRN estavam inseridos num serviço de hospital-dia, com características de atendimento cooperativo, também denominado multidisciplinar, desenvolvido no Centro de Medicina do Idoso (CMI) do Hospital Universitário de Brasília (HUB).

De um total de sete grupos atendidos no CMI, foram selecionados 21 pacientes que cumpriram todos os referidos critérios, sendo 11 homens e 10 mulheres, com média de idade respectivamente de 77,0 (5,9) e 72,8
$(6,4)$ anos. Na média total, o grupo apresentava 75,0 (6,4) anos de idade, com escolaridade de 8,9 $(4,0)$ anos. 0 propósito do estudo foi explicado aos participantes e a seus familiares ou cuidadores, tendo sido eles recrutados somente após o livre aceite da participação, atestado pela assinatura do termo de consentimento livre e esclarecido.

Para análise dos efeitos deste PRN, os critérios de inclusão foram: diagnóstico compatível com demência instalada; participação em todas as seis sessões da oficina de memória de jardinagem e pistas coloridas - OEM; realização das duas sessões de AN, completando assim as oito semanas de trabalho do PRN.

Todos os participantes faziam uso de um ativador da memória anticolinesterásico, com tempo de uso de 4, 12 ou 30 semanas antes da OEM (Tabela 1).

\section{Procedimentos}

\section{Avaliação Neuropsicológica}

Uma bateria neuropsicológica, organizada pelo Serviço de Neuropsicologia do CMI, foi aplicada previamente e ao fim da oficina de memória, representado a primeira e a oitava sessão do PRN. As avaliações examinaram o impacto da oficina de memória sobre as funções cognitivas, como memória, fluência verbal, motricidade visuoconstrutiva e comportamentos referentes à depressão. Essa bateria foi composta pelos seguintes testes neuropsicológicos: Miniexame do Estado Mental (Mini-Mental State Examination - MEEM) (M.F. Folstein, S.E. Folstein \& McHugh, 1975), Teste do Desenho Livre de um Relógio (DRE) (Wolf-Klein, Silverstone, Levy \& Brod, 1989), Escala de Depressão Ge-riátrica (EDG) (Yesavage et al., 1983), Teste de Fluência Verbal para palavras iniciadas em F, A e S (FAS-FV) (Bayles \& Kasniak, 1987), Teste de Fluência Verbal Semântica para palavras de categorias como nomes de animais (FAS-FS) (Bayles \& Kasniak, 1987), Subteste Verbal de Semelhanças da Escala de Wechsler de Inteligência Adulta (SE-WAIS) (Nascimento, 2004) e Escala de Memória de Wechsler (WMS) (Wechsler, 1987), composta pelos Subtestes de Informação e Orientação (IN-OR), Reprodução Direta de Dígitos (DD), Reprodução Inversa de Dígitos (DI), Reprodução de Memória Lógica Imediata (ML1), Reprodução 
de Memória Lógica Tardia após 30 minutos (ML2), Aprendizagem de Pares de Fácil Associação para Evocação Imediata (PAF1), para Evocação Tardia (PAF2), Aprendizagem de Pares de Difícil Associação para Evocação Imediata (PAD1) e para Evocação Tardia após 30 minutos (PAD2).

\section{Oficina de jardinagem e pistas coloridas para estimulação da memória}

O trabalho sistemático de oficina envolveu seis sessões em grupo, com duração de duas horas, uma vez por semana. Foi monitorado o desempenho de cada idoso nas atividades propostas, através de observação direta, mediante anotações feitas pelos examinadores. As atividades foram divididas nos seguintes tópicos:

Primeira sessão (escolha do sinalizador e associação com uma reminiscência): A oficina iniciou-se com a escolha do sinalizador, ou seja, cada participante escolheu a cor de seu avental, a ser utilizado em todas as sessões, sendo que havia uma cor diferenciada para cada um deles. A escolha do sinalizador deu-se por meio de uma discussão em grupo, em que o participante associava a cor escolhida a um significado suscitado por suas reminiscências históricas e emocionais. Assim, uma das participantes concluiu que escolheu o sinalizador amarelo por ser a "cor do ouro" e a "cor de sua aliança de casamento". Ela se sentia muito orgulhosa

Tabela 1. Descrição dos quadros patológicos demenciais de Alzheimer (DA) e Vascular (VAS) e dos medicamentos administrados antes do PRN ordenando os pacientes em 3 grupos de tratamento farmacológico (4, 12, e mais de 30 semanas) e desempenhos desses pacientes e médias dos grupos obtidos na Escala de Depressão Geriátrica de Yesavage. Brasília (DF), 2006-2009.

\begin{tabular}{|c|c|c|c|c|}
\hline Doenças & Fármacos ativadores da Memória & $\begin{array}{c}\text { Semanas de uso dos fármacos Ativadores } \\
\text { da Memória antes do PRN }\end{array}$ & EDG antes do PRN & EDG após o PRN \\
\hline DA & Rivastigmina & 4 & 14 & 5 \\
\hline VAS & Rivastigmina & 4 & 13 & 4 \\
\hline DA & Donepezila & 4 & 10 & 5 \\
\hline DA & Rivastigmina & 4 & 7 & 5 \\
\hline VAS & Donepezila & 4 & 5 & 5 \\
\hline DA & Rivastigmina & 4 & 5 & 5 \\
\hline VAS & Rivastigmina & 4 & 4 & 5 \\
\hline DA & Donepezila & 4 & 5 & 4 \\
\hline DA & Galantamina Donepezila & 4 & 5 & 3 \\
\hline \multirow[t]{3}{*}{ DA } & Rivastigmina & 4 & 5 & 2 \\
\hline & Média (DP) & & $7,3(3,7)$ & $4,3^{*}(1,0)$ \\
\hline & Wilcoxon & & $Z=-2,31$ & $p<0,02$ \\
\hline VAS & Donepezila Memantina & 12 & 7 & 5 \\
\hline DA & Rivastigmina & 12 & 7 & 4 \\
\hline DA & Donepezila & 12 & 5 & 2 \\
\hline VAS & Rivastigmina & 12 & 5 & 1 \\
\hline DA & Rivastigmina & 12 & 5 & 1 \\
\hline \multirow[t]{3}{*}{ VAS } & Rivastigmina & 12 & 3 & 2 \\
\hline & Média (DP) & & $5,3(1,5)$ & $2,5^{*}(1,6)$ \\
\hline & Wilcoxon & & $Z=-2,21$ & $p<0,02$ \\
\hline VAS & Rivastigmina & 30 & 12 & 14 \\
\hline DA & Rivastigmina Memantina & 30 & 10 & 10 \\
\hline DA & Rivastigmina & 30 & 4 & 5 \\
\hline VAS & Rivastigmina & 30 & 4 & 5 \\
\hline \multirow[t]{3}{*}{ VAS } & Rivastigmina & 30 & 5 & 2 \\
\hline & Média (DP) & & $7,0(3,7)$ & $7,2(4,7)$ \\
\hline & Wilcoxon & & $Z=-0,36$ & $p=0,71$ \\
\hline
\end{tabular}

* Os valores indicados são significativos entre os grupos $(p<0,05)$ após a reabilitação, conforme Wilcoxon.

DA: demenciais de Alzheimer; VAS: vascular; PRN: programas de reabilitação neuropsicológica; EDG: escala de depressão geriátrica de Yesavage; DP:

234

desvio-padrão. 
por manter um casamento de mais de 50 anos (reminiscência) e, por isso, passou a associar o sinalizador "cor amarela" com a "cor de sua aliança", ligando-a a sua reminiscência "casamento de 50 anos". Assim, diante da solicitação de recordar a cor do seu avental em cada sessão, a paciente chegou a evocar: "a cor do ouro, a cor da minha aliança, a cor do meu avental é o amarelo." Outro participante preferiu a cor azul, que passou então a ser o seu sinalizador. Isso porque sua reminiscência associada foi ter sido torcedor apaixonado de um time de futebol identificado por aquela cor. Desse modo, nos exemplos citados, "o amarelo relacionado ao ouro da aliança" teve como dica contextual de reminiscência que "a sua cor tem a ver com a joia que lembra a história de seu casamento feliz", enquanto para o "azul relacionado à cor da camisa do time por quem o paciente torcia", a dica contextual foi "você escolheu a cor do time do seu coração". Dessa maneira, todos os participantes escolheram a cor de seu avental dentre as doze ofertadas, ou seja, elegeram o sinalizador que passaram a utilizar nas três sessões seguintes, assim treinando a associação cor/significado, pela evocação de uma reminiscência marcante do ponto de vista histórico e/ou emocional.

Segunda sessão (pintura do vaso): Inicialmente foi feita a recuperação livre da escolha do SI e seu significado de reminiscência. Em seguida, cada participante realizou a pintura de um vaso, utilizando a cor do sinalizador.

Terceira sessão (escolha da muda, preparação do vaso e plantio): Novamente foi realizada a recuperação livre do sinalizador e de seu significado de reminiscência, além do reconhecimento do vaso pintado na cor do respectivo sinalizador. Em seguida, foram ofertados aos participantes 6 a 8 tipos diferentes de mudas aromáticas, conforme o número de participantes no grupo. Cada um deles realizava livremente sua escolha, conforme suas reminiscência e preferências. Por fim, foi feito o plantio da muda no vaso sinalizador.

Quarta sessão (reconhecimento do vaso sinalizador com a muda plantada e rega da planta): Foi realizada novamente a recuperação livre do avental sinalizador, bem como o reconhecimento do vaso sinalizador de cada participante, já com as mudas plantadas. Nessa sessão os participantes regaram as suas mudas, cuidaram delas e levaram-nas para casa.
Quinta sessão: Foi realizada, pela última veze individualmente, a recuperação livre do sinalizador do avental e seu significado de reminiscência. No término da sessão, foi observado o desempenho final dos participantes sobre a aprendizagem dos sinalizadores. Assim, registrou-se: a) quais idosos aprenderam a evocar livremente, sem ajuda, o nome do sinalizador (cor) que havia escolhido e a reminiscência associada a essa escolha; b) quais idosos aprenderam a evocar o sinalizador (cor) correto somente com a apresentação da sua reminiscência; e c) quais idosos não conseguiram evocar corretamente o seu sinalizador (cor), mesmo com apresentação da reminiscência. O critério de aprendizagem foi tomado pelas respostas emitidas nessa última sessão, ou seja, após o treino das quatro sessões anteriores.

Sexta sessão (passeio): Foi realizado um passeio em áreas públicas com os idosos, seus cuidadores e a equipe interdisciplinar. O objetivo foi integrar os idosos e seus familiares numa situação de enfrentamento, com discussões finais sobre os benefícios do grupo psicoeducativo.

\section{Resultados}

O teste não paramétrico de Wilcoxon revelou aumento significativo dos escores obtidos depois do PRN no MEEM $[(Z=-1,98) p<0,05]$, no Subteste Verbal de Semelhanças da Escala Wechsler de Inteligência Adulta $[(Z=-2,09) p<0,05]$ e no Subteste Aprendizagem de Pares de Fácil Associação para Evocação Tardia da Escala de Memória de Wechsler $[(Z=-2,07) p<0,05]$. Revelou, ainda, redução significativa dos escores de depressão avaliados na Escala de Depressão Geriátrica de Yesavage $[(Z=-3,02)$ $\mathrm{p}<0,00]$, como se observa na Tabela 2 . Os demais testes aplicados não apresentaram diferenças significativas.

No intuito de entender as relações entre melhora de desempenho cognitivo e redução de indicadores de depressão, foram realizadas outras análises não paramétricas. Primeiro, foi comparado o impacto do PRN em três faixas etárias (de 55 a 70 anos, de 71 a 77, e de 78 ou mais) em relação ao desempenho nos testes anteriores e posteriores ao PRN. A seguir, foram comparadas três faixas de escolaridade (de 0 a 4 anos de escolaridade, de 8 a 11, e de 12 ou mais) em relação ao desempenho nos testes anteriores e posteriores ao PRN. Finalmente, foram comparados os diferentes tipos de demência 
(Alzheimer e Vascular) em relação ao desempenho nos testes anteriores e posteriores ao PRN.

Não foi encontrada nenhuma diferença significativa entre o desempenho nos testes anteriores e posteriores ao PRN, em nenhum dos grupos ( $p>0,05$ ), conforme o teste de Wilcoxon. Entretanto, quando os pacientes foram classificados conforme o tempo de administração de fármacos para melhora da memória antes da inclusão no PRN (grupo de 4, 12 ou 30 semanas), o teste revelou diferença significativa no desempenho da EDG antes e após o PRN apenas nos grupos de 4 e 12 semanas de tratamento [respectivamente $(Z=-2,31)$ $\mathrm{p}<0,02$ e $(Z=-2,21) \mathrm{p}<0,02]$.

Porém, o mesmo não ocorreu no grupo que vinha tomando a medicação há mais de 30 semanas
$[(Z=-0,36) p=0,71$, quando de sua inclusão no PRN, como se pode observar na Tabela 1.

A análise não paramétrica de Wilcoxon apontou ainda diferenças significativas no que se refere ao desempenho na EDG, antes e depois do PRN, nos grupos de participantes que necessitavam de dica contextual (reminiscência para evocar o nome correto do sinalizador) $[(Z=-2,08) p<0,05]$ e nos participantes que evocavam corretamente os sinalizadores e reminiscências por livre recordação $[(Z=-1,89) p<0,05]$. O mesmo não ocorreu no grupo dos participantes que não conseguiram evocar seus sinalizadores corretamente, mesmo com a apresentação da dica, ou seja, da reminiscência associada $[(Z=-1,06) p=0,10]$ como se pode observar na Tabela 3. Pode-se observar, ainda, que os idosos que necessitaram

Tabela 2. Pacientes idosos demenciados atendidos pelo da Memória ordenados pelos anos de estudo e seus desempenhos em testes neuropsicológicos antes e após o Programas de Reabilitação Neuropsicológica com as referências de ponto total e ponto de corte. Brasília (DF), 2006-2009.

\begin{tabular}{|c|c|c|c|c|c|c|c|c|c|c|c|c|c|c|}
\hline & & $\mathrm{Te}$ & eur & icolć & $\cos a$ & ados & os pac & entes pr & é pó & reabili & ção n€ & ropsic & lógica & \\
\hline & MEEM & $D R E$ & $F F$ & FS & SEM & $D D$ & $D /$ & $I N-O R$ & $C M$ & $M L 1$ & $M L 2$ & PAF1 & PAF2 & $E D G$ \\
\hline Média (antes PRN) & 19,0 & 4,6 & 22,7 & 8,8 & 4,6 & 6,2 & 3,0 & 10,3 & 2,8 & 5,0 & 1,5 & 5,2 & 1,7 & 6,6 \\
\hline DP & 4,6 & 2,6 & 11,6 & 5,5 & 3,6 & 2,2 & 1,6 & 3,1 & 1,6 & 3,9 & 3,4 & 1,1 & 1,1 & 3,8 \\
\hline Média (após PRN) & $20,3^{*}$ & 4,7 & 21,5 & 9,9 & $6,3^{*}$ & 6,9 & 3,4 & 10,3 & 3,1 & 6,3 & 1,5 & 5,8 & $2,0^{*}$ & $4,4^{*}$ \\
\hline DP & 3,9 & 2,9 & 14,1 & 6,6 & 4,4 & 2,1 & 1,8 & 3,3 & 2,1 & 3,9 & 2,8 & 1,2 & 1,2 & 3,1 \\
\hline Teste Z Wilcoxon & $-1,98$ & $-0,47$ & $-0,56$ & $-0,57$ & $-2,09$ & $-0,80$ & $-1,39$ & $-0,36$ & $-1,12$ & $-1,87$ & $-0,10$ & $-1,27$ & $-2,07$ & $-3,02$ \\
\hline Significância (p) & $<0,05$ & 0,63 & 0,57 & 0,56 & $<0,05$ & 0,42 & 0,16 & 0,71 & 0,26 & 0,06 & 0,91 & 0,20 & $<0,05$ & $<0,00$ \\
\hline
\end{tabular}

" $p<0,05$ conforme o Teste de Wilcoxon para as médias significativamente diferentes após o PRN. MEEM: miniexame do estado mental; DRE: teste do desenho do relógio; FF: teste de fluência fonêmica; FS: teste de fluência semântica; SEM: sub-teste de semelhanças da (escala de inteligência adulta de Wechsler); DD: sub-teste de repetição de dígitos diretos da WAIS; DI: sub-teste de repetição de dígitos indiretos da WAIS; IN-OR: sub-teste de informação e orientação da WMS (Escala de Memória Wechsler); CM: sub-teste controle mental da WMS; ML1: sub-teste de memória lógica imediata da WMS; ML2: sub-teste de memória lógica tardia da WMS; PAF1: sub-teste de aprendizagem imediata de pares de fácil associação da WMS; PAF2: sub-teste de aprendizagem tardia de pares de fácil associação da WMS; EDG: escala de depressão geriátrica de Yesavage.

Tabela 3. Desempenho na EDG em relação ao aprendizado na tarefa de reabilitação neuropsicológica: resposta associada Sinalizador - Reminiscência. Brasília (DF), 2006-2009.

\begin{tabular}{|c|c|c|c|}
\hline & \multicolumn{2}{|l|}{ EDG antes do PRN } & \multirow[t]{2}{*}{ EDG após o PRN } \\
\hline & Pacientes que evocaram por livre recordação a tarefa $\mathrm{RN}(\mathrm{N}=7)$ & & \\
\hline \multirow{8}{*}{ 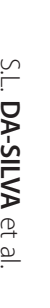 } & Média & $4,8(1,2)$ & $3,2^{*}(1,7)$ \\
\hline & Wilcoxon & $Z=-1,89$ & $p<0,05$ \\
\hline & Pacientes que evocaram com dica da reminiscência a Tarefa RN (N=10) & & \\
\hline & Média & $8,7(3,6)$ & $5,8^{*}(3,5)$ \\
\hline & Wilcoxon & $Z=-2,08$ & $p<0,05$ \\
\hline & Pacientes que não evocaram resposta da Tarefa $\mathrm{RN}(\mathrm{N}=4)$ & & \\
\hline & Média & $4,7(0,5)$ & $3,2(2,0)$ \\
\hline & Wilcoxon & $Z=-1,06$ & $p=0,10$ \\
\hline
\end{tabular}


da apresentação da reminiscência para evocar corretamente o sinalizador eram os que possuíam os maiores escores de depressão antes do PRN. Porém, depois da intervenção, foram os que mais reduziram esses escores.

No grupo psicoeducativo, os relatos dos familiares traduziram impressão de alterações positivas nos idosos, referentes a aspectos sociais, como a melhora do relacionamento familiar, e ao aumento da autoestima, além de alguns relatos de melhora de orientação durante as sessões de estimulação cognitiva, principalmente diante da autopercepção positiva de seu desempenho e do resultado de sua obra final na oficina (o vaso pintado, com a muda plantada). No que se refere aos cuidadores, os relatos do grupo evidenciaram uma melhor aceitação das limitações do idoso, bem como a adesão dos familiares ao tratamento.

\section{Discussão}

Os dados demonstram que alguns idosos estabilizaram défices, ou os suprimiram, mas também acentuaram ou expressaram novos défices. Porém, de certa forma, pode-se dizer que o PRN foi significativo em relação à estimulação da memória, já que apenas 4 dos 21 idosos estudados não conseguiram aprender a associação entre a reminiscência significativa e seu sinalizador, até a 5a sessão da oficina.

Considerando-se os 17 idosos que conseguiram memorizar seus sinalizadores, pode-se inicialmente inferir que, durante a oficina, foi construída uma conexão de fatos, percepções e significados sobre uma reminiscência associada à escolha de um sinalizador, o que foi estimulado a cada sessão durante o desenvolvimento da oficina. Essas conexões provavelmente estavam sendo estimuladas quando o idoso era lembrado de que havia escolhido um sinalizador, em função de uma reminiscência vivida por ele. Se não ocorresse a recordação livre, a reminiscência associada era imediatamente apresentada como dica para evocação do sinalizador, de forma que, em cada sessão, ocorria a estimulação de uma rede de informações sobre o sinalizador, ligando episódios remotos particulares de cada participante aos eventos semanais presentes na oficina. Assim, a primeira sessão consistiu na escolha do avental da cor sinalizadora; a segunda, na pintura do vaso com esse sinalizador; a terceira, no reconhecimento do vaso pintado com a cor sinalizadora, seguido de plantio; a quarta, no reconhecimento do vaso sinalizador com a muda plantada pelo participante. Esses significados sobre a cor, avental e vaso - ou, tecnicamente, sobre o sinalizador - possivelmente foram armazenados de forma agrupada, o que facilitou a evocação do sinalizador correto pela maioria dos participantes.

O PRN aplicado neste estudo, de certa forma, está em conformidade com as ideias de Baddeley (1998), segundo as quais palavras distintas que expressem conceitos semelhantes possuem grande possibilidade de serem armazenadas de forma agrupada. O autor explica o armazenamento da memória semântica a partir do efeito de facilitação da recordação, propiciada por palavras distintas com significados comuns, de tal modo que a exposição a uma palavra promoveria uma ativação espraiada da rede semântica.

O uso de associação semântica, como estratégia de reabilitação neuropsicológica, tem sido discutido em vários artigos da área. De forma geral, ela é capaz de expandir as associações entre informações aprendidas ou armazenadas. A alteração do padrão de curva de posição serial, pela introdução de palavras semanticamente relacionadas entre si nas posições intermediárias das listas, produz um novo pico de recordações corretas. Esse efeito de facilitação já foi observado em portadores de Alzheimer em fase inicial (Abrisqueta-Gomez, 1999) e em pacientes pós-cirúrgicos de aneurisma cerebral (Da Silva, Huang, Tin \& Bueno, 2006).

Assim, os resultados deste estudo permitem concluir que a associação sistemática de um sinalizador (cor, pintura, dança, jardinagem ou outra temática) a reminiscências aponta as conexões de redes de informação. Tais conexões se dão não somente no nível das palavras, mas envolvem também fatos recentemente vivenciados nas oficinas de memória, de modo que conceitos semânticos podem associar-se a sinalizadores, por meio da recuperação de reminiscências preservadas e emocionalmente significativas.

Esta proposta de oficina de memória está também de acordo com Wilson (1997), que enfatiza que, independentemente da dificuldade em se recuperar plenamente a memória de pacientes com distúrbios amnésicos orgânicos, não se deve deixar de buscar maneiras de melhorar seu bem-estar e qualidade de vida. 
Ademais, a redução dos indicadores de depressão após o PRN, verificada no presente estudo, está diretamente de acordo com os dados obtidos por Abrisqueta-Gomez (2006), que demonstrou a redução desses escores após PRN, através da Escala de Listagem Revisada de Problemas de Memória e Comportamento (LRPMC).

A neuropsicologia tem se preocupado em desenvolver reabilitações neuropsicológicas que abracem situações similares às desenvolvidas nas assistências de saúde, principalmente no que se refere às atividades sociais e recreativas. No entanto, nem sempre estão passíveis de monitoramento cognitivo. É ilimitado o ganho de inovações em tecnologias de saúde que buscam integrar a pesquisa básica em neurociências sobre a reabilitação cognitiva em idosos com situações ambientais e clínicas envolvendo estimulações da memória, do humor, da sociabilidade, do lazer e da interação familiar com monitoramento de variáveis (biológicas, psicológicas e ambientais).

As metas principais, no início do século XXI, devem incluir o atendimento às doenças do envelhecimento e a inclusão social do idoso como forma preventiva da saúde mental. Por conseguinte, almeja-se maior desenvolvimento de estratégias cognitivas para compensar as dificuldades do paciente idoso, evitando situações que levem a sua depreciação e desvalorização, bem como na promoção de orientações de realidade, sociabilidade, melhora do humor, prazer e qualidade de vida. Vários pesquisadores, como Abrisqueta-Gomez et al. (2002), Ávila et al. (2004), Bottino, Carvalho e Alvarez (2002), Da Silva (2004), De Vreese et al. (2001), Prigatano (1995), Wilson (1987; 1997), vêm apoiando esse tipo de inovação tecnológica e preocupação no atendimento à saúde do idoso.

Aárea de saúde vem afirmando, atualmente, que a efetividade do tratamento para doenças crônicas degenerativas está atrelada à ação de equipes cooperativas e/ou multidisciplinares (Abrisqueta-Gomez, 2002; Bottino et al., 2002; Da-Silva, 2004), pois nessa condição é mais viável construir programas de intervenção e educação, que considerem um maior número possível de variáveis envolvidas no adequado atendimento aos pacientes e seus familiares. Nesse sentido, estratégias cooperativas podem facilitar o enfrentamento e a com238 preensão da doença, bem como a adesão ao tratamento, melhorando a qualidade de vida do paciente e da família (Kerns, 1995).

Assim, é importante ressalvar que os resultados desse estudo possivelmente estão ligados não somente ao PRN analisado, mas também a um esforço cooperativo das demais equipes que atenderam os participantes durante todo o programa de tratamento no hospital-dia do Centro de Medicina do Idoso, além da adesão dos pacientes e familiares, facilitada pelo grupo de psicoeducação.

De modo similar ao presente estudo, Kurz et al. (2009) apresentaram uma análise dos efeitos de um PRN, que repercutiu em melhora no desempenho em testes neuropsicológicos de sujeitos atendidos por equipe multidisciplinar. Dessa forma, tendo em vista os resultados obtidos neste estudo, pode-se apontar que as análises devem ser consideradas dentro dos respectivos limites, ou seja, um PRN ligado a treino de memória tem maior probabilidade de promover alterações cognitivas se o programa estiver associado a um atendimento multidisciplinar.

\section{Considerações Finais}

Os pacientes demenciados em estágio inicial ou intermediário tiveram melhora cognitiva significativa no teste de rastreio do MEEM e nos testes de memória verbal declarativa, como Subteste Verbal de Semelhanças da Escala Wechsler e Subteste de Aprendizagem de Pares de Fácil Associação para Evocação Tardia da Escala de Memória de Wechsler. Apresentaram, ainda, redução dos indicadores de Depressão da EDG, corroborando os dados de Kurz et al. (2009), segundo os quais pacientes com demência leve, após PRN com treino cognitivo, tiveram melhora significativa nos testes de memória declarativa episódica verbal California Verbal Learning Test e redução nos escores de depressão do Inventário de Depressão de Beck. A melhora do estado de humor, representada pela redução dos escores de depressão obtidos na EDG neste estudo, está em conformidade com os resultados de Belleville et al. (2006), Rapp et al. (2002), Rozzini et al. (2006) e Talassi et al. (2007), os quais também relataram melhora cognitiva, de humor e bem-estar psíquico, em grupos de pacientes idosos com declínio cognitivo e demência em estágio inicial. 


\section{Referências}

Abrisqueta-Gomez, J. (1999). Avaliação neuropsicológica nas fases inicial e moderada da demência do tipo Alzheimer. Tese de doutorado não-publicada, Universidade Federal de São Paulo.

Abrisqueta-Gomez, J. (2006). Relevância das intervenções neuropsicológicas nas alterações de humor e comportamento no idoso. In D.V.S Falcão \& C.M.S. Brito Dias (Orgs.), Maturidade evelhice (Vol. 2, pp.407-422). São Paulo: Casa do Psicólogo.

Abrisqueta-Gomez, J., Brucki, S. M. D., Canali, F., Oliveira, E., Ponce, C., Vieira, V., et al. (2002). Neuropsychological rehabilitation program in cognitive impairment and dementia. In L. Battistin, M. Dam \& P. Tonin (Orgs.), Proceedings of the $3^{\text {rd }}$ World Congress Neurological Rehabilitation (pp.399-407). Venice: Monduzzi Editore.

Ávila, R., Bottino, C. M. C., Carvalho, I. A. M., Santos, C. B., Seral, C., \& Miotto, E. C. (2004). Neuropsychological rehabilitation of memory deficits and activities of daily living in patients with Alzheimer's disease: a pilot study. Brazilian Journal of Medicine and Biological Research, 37 (11), 1721-1729.

Baddeley, A. D. (1998). Human memory: theory and pratice. Boston: Allyn \& Bacon.

Bayles, K. A., \& Kasniak, A. (1987). Communication and cognition in normal aging. Boston: College-Hill.

Belleville, S., Gilbert, B., \& Fontaine, F. (2006). Improvement of episodic memory in persons with mild cognitive pmairment and healthy older adults: evidence from a cognitive intervention program. Demential Geriatric Cognitive Disorder, 22, 486-499.

Bottino, C. M., Carvalho, I. A., \& Alvarez, A. M. (2002). Cognitive rehabilitation in Alzheimer's disease patients: multidisciplinary team report. Arquivos de Neuropsiquiatria, 60 (1), 70-79.

Bourgeois, M. S. (1990). Enhancing conversation skills in patients with Alzheimer's disease using a prosthetic memory aid. Journal of Applied Behaviour Analysis, 23 (1), 29-42.

Brooks, D. N., \& Baddeley, A. (1976). What can amnesic patients learn. Neuropsychologia, 14 (1),111-2.

Cipriani G., Bianchetti A., \& Trabucchi M. (2006). Outcomes of a computer-based cognitive rehabilitation program on alzheimer's disease patients compared with those on patients affected by mild cognitive impairment. Archives of Gerontological Geriatric, 43 (3), 327-335.

Da-Silva, S. L. (2004). Reabilitação neuropsicológica em idosos: uma gota no oceano. Revista Eletrônica ComCiência, Maio LaborJor-Unicamp-SBPC. Recuperado em março 11, 2004, disponível em <http://www.comciencia.br/ reportagens/memoria/16.shtmls.

Da-Silva, S. L., Huang, Tin Po., \& Bueno, O. F. A. (2006). Free recall facilitation in cerebral aneurysm surgery patients. Neurobiologia, 69 (1), 22-29.

De Vreese, L. P., Néri, M., Fioravanti, M., Belloi, L., \& Zanetti, O. (2001). Memory rehabilitation in Alzheimer's disease: a review of progress. International Journal of Geriatric Psychiatry, 16 (8), 794-809.

Folstein, M. F., Folstein, S. E., \& McHugh, P. R. (1975). Mini mental state: a pratical method for grading the cognitive state of patients for the clinician. Journal of Psychiatric Research, 12 (3), 189-198.

Fraser, M. (1992). Memory clinics and memory training. In $\mathrm{T}$. Arie (Org), Recent advances in psychogeriatrics (pp.105-115). London: Churchill Livingstone.

Garrido, R., \& Almeida, O. P. (1999). Distúrbios de comportamento em pacientes com demência. Impacto na vida do cuidador. Arquivos de Neuropsiquiatria, 57 (2-B), 427-434.

Grady, C. L., Haxby, J. V., Horwitz, B., Berg, G., \& Rapoport, S. I. (1988). Neuropsychological and cerebral metabolic function in early vs late onset dementia of the Alzheimer type. Neuropsychologia, 25, 807-816.

Jankowsky, J. L., Melnikova, T., Fadale, D. J., Xu, G. M., Slunt, H. H., \& Gonzales, V. (2005) Environmental enrichment mitigates cognitive deficits in a mouse Model of Alzheimer's disease. The Journal of Neuroscience, 25 (21), 5217-5224.

Joca, S. R. L., Padovan, C. M., \& Guimarães, F. S. (2003). Estresse, depressão e hipocampo. Revista Brasileira de Psiquiatria, 25 (Supl II), 46-51.

Kerns, R. D. (1995). Family assesment and intervention. In P.M. Nicassio \& T.W. Smith (Orgs.), Managing chronic illness:abiopsychosocial perspective. Washington: American Psychological Association.

Kolb, B., \& Gibb, R. (2008). Principles of neuroplasticity and behavior. In D.T Stuss, G. Winocur \& I.H. Roberston (Orgs.), Cognitive neurorehabilitation: evidence and application. Cambridge: University Press.

Kurz, A., Pohl, C., Ramsenthaler, M., \& Sorg, C. (2009). Cognitive rehabilitation in patients with mild cognitive impairment. International Journal of Geriatrical Psychiatry, $24(2), 163-168$.

Lincoln, N., \& Nair, R. (2008). Outcome measurement in cognitive neurorehabilitation. In D. T. Stuss, G. Winocur \& I. H. Roberston (Orgs.), Cognitive neurorehabilitation: evidence and application. Cambridge: University Press.

Londos E., Boschian K., Lindén A., Persson, C., Minthon, L., \& Lexell, J. (2008). Effects of a goaloriented rehabilitation program in mild cognitive impairment: a pilot study. Journal of Alzheimer's Disease and Other Dementias, 23 (2), 177-183.

Nascimento, E. (2004). Adaptação, validação e normatização do WAIS-III para uma amostra brasileira. In D. Wechsler (Org.), WAIS-III: manual para administração e avaliação (Vol. 1, pp.161-192). São Paulo: Casa do Psicólogo.

Prigatano, G. P. (1995). Personality and social aspects of memory rehabilitation. In A. D. Baddeley, B. A. Wilson \& F.N. Watts (Orgs.), Handbook of memory disorders (pp.603-614). Chichester: John Wiley.

Rapp S., Brenes G., \& Marsh, A. P. (2002). Memory enhancement training for older adults with mild cognitive impairment: a preliminary study. Aging Mental Health, 6, 5-11.

Rodrigues, A. D., \& Rothi, L. J. G. (2008). Principles in conducting rehabilitation research. In D. T. Stuss, G. Winocur \& I. H. 
Roberston (Orgs.), Cognitive neurorehabilitation: evidence and application. Cambridge: University Press.

Rosenzweig, M. R., Krech, D., Bennett, E. L., \& Diamond, M. (1962). Effects of environmental complexity and tranining on brain chemistry and anatomy: a replication and extension. Journal of Comparative and Physiological Psychology, 55 (4), 429-437.

Rozzini, L., Costardi, D., \& Vicini-Chilovi, B. (2006). Efficacy of cognitive rehabilitation in patients with mild cognitive impairment treated with cholinesterase inhibitors. International Journal of Geriatric Psychiatry, 22 (4), 356-360.

Sohlberg, M. M., \& Mateer, C. A. (2001). Cognitive rehabilitation: an integrative neuropsychological approach. New York: Guilford.

Squire, L. R. (1987). Memory and brain. Oxford, NY: University Press.

Talassi, E., Guerreschi, M., Feriani, M., Fedi, V., Bianchetti, A., \& Trabucchi M. (2007). Effectiveness of a cognitive rehabilitation probram in mild dementia (MD) and mild cognitive impairment (MCl): a case control study. Archives of Gerontological Geriatric, 44 (Suppl 1), 391-399.

Vaisman, H., Almeida, K. M. H., \& Almeida, O. P. (1997). Abordagens psicoterápicas para idosos demenciados. In O. Forlenza \& O. P. Almeida (Orgs.), Depressão e demência no idoso (pp.167-192). São Paulo: Lemos.
Wechsler, D. (1987). Wechsler memory scale: revised manual. San Antonio: Psychological Corporation.

Wilson, B. A. (1987). Rehabilitation of memory. New York: Guilford.

Wilson, B. A. (1997). Cognitive rehabilitation: how it is and how it might be (Critical review). JINS, 3, 487-496.

Wilson, B. A. (2009). Memory rehabilitation: integrating theory and practice. New York: Guilford.

Wilson, B. A., \& Moffat, N. (1992). The development of group memory therapy. In B. A. Wilson \& N. Moffat. (Eds.), Clinical management of memory problems ( $2^{\text {nd }}$ ed., pp.243-73). London: Chapman and Hall.

Wolf-Klein, G. P., Silverstone, F. A., Levy, A. P., \& Brod, M. S. (1989). Screening for Alzheimer's disease by clock drawing. Journal of American Geriatric Society 37(8): 730-734.

Yesavage J. A., Brink, T.L., Rose, T.L., Lum, O. Huang, V., Adey, M.B., et al (1983). Development and validation of a geriatric depression screening scale: a preliminary report. Journal of Psychiatric Research, 17 (1), 37-49.

Recebido em: 2/4/2009

Versão final reapresentada em: 9/2/2010

Aprovado em: 11/3/2011 